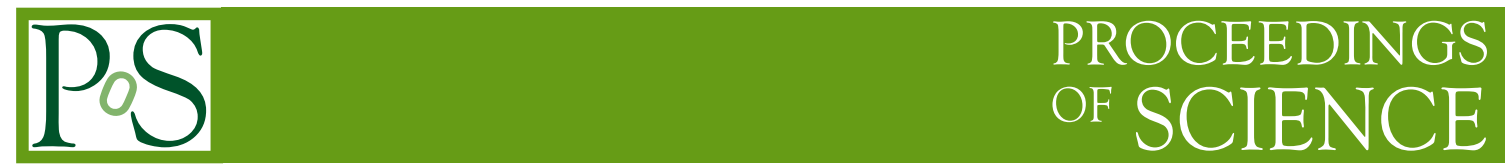

\title{
SUSY overview at ATLAS
}

\section{Evgeny Khramov*}

Joint Institute for Nuclear Research (JINR), Dubna, Russia

On behalf of the ATLAS Collaboration

E-mail: Evgeniy.Khramov@cern.ch

This contribution summarises recent results on searches for squarks and gluinos, as well as the electro-weak production of sleptons, charginos and neutralinos. The searches are performed on more than $20 \mathrm{fb}^{-1}$ of $\sqrt{s}=8 \mathrm{TeV}$ proton-proton collision 2012 data recorded with the ATLAS detector at the Large Hadron Collider. No significant excess has been found in any of the searches. These results are interpreted in the framework of various Supersymmetry models.

The XXI International Workshop High Energy Physics and Quantum Field Theory,

June 23 - June 30, 2013

Saint Petersburg Area, Russia

${ }^{*}$ Speaker. 


\section{Introduction}

Supersymmetry (SUSY) is one of the most popular candidate for physics beyond the Standard Model (SM). It predicts the a supersymmetric partner for each SM particle that differs in spin by 1/2 from its SM counterpart. Supersymmetry provides solutions for some Standard Model (SM) problems as the hierarchy problem, unification of gauge couplings. In case of R-parity $(R=$ $(-1)^{3 \cdot(B-L)+2 S}$ ) conservation SUSY particles are produced in pairs and a weakly interacting lightest supersymmetric particle (LSP) is stable and is a candidate for the Dark Matter (DM). The two produced LSP in each SUSY event escape detection, thus generating an imbalance in transverse momentum ( $p_{\mathrm{T}}^{\text {miss }}$ and its magnitude $E_{\mathrm{T}}^{\text {miss }}$ ) which is the most characteristic signature for SUSY.

First, a summary of inclusive searches for gluino, $\tilde{g}$, and first two generations of squarks, $\tilde{q}$, production are presented in this note. Analyses with different final states with large missing transverse momentum, different jet and lepton multiplicities are discussed.

A study of the expected SUSY particle spectrum derived from naturalness considerations suggests that the supersymmetric partners of the third generation SM quarks are the lightest coloured supersymmetric particles. This may lead to the lightest bottom squark (sbottom, $\tilde{b}_{1}$ ) and top squark (stop, $\tilde{t}_{1}$ ) mass eigenstates being significantly lighter than the other squarks and the gluinos. As a consequence, $\tilde{b}_{1}$ and $\tilde{t}_{1}$ could be produced with relatively large cross sections at the Large Hadron Collider (LHC). The recent results with two jets originating from the hadronisation of the b-quarks and large $E_{\mathrm{T}}^{\text {miss }}$ as well as one isolated electron or muon, jets, and missing transverse momentum are summarized.

If the masses of the gluinos and squarks are large, the direct production of charginos, neutralinos, and sleptons may dominate the production of SUSY particles at the Large Hadron Collider. Searches for final states with two oppositely-charged leptons, three leptons (electrons or muons) or at least four isolated (electrons, muons and leptonic tau decays) and $E_{\mathrm{T}}^{\text {miss }}$, final states with two hadronically decaying taus are described.

The analysis results are interpreted in the context of sevearl SUSY models, such as simplified supersymmetric models, GMSB and mSUGRA/CMSSM models using proton-proton collision data at a centre of mass energy of $8 \mathrm{TeV}$ recorded with the ATLAS detector [1] at the LHC. The theory references can be found in the corresponding ATLAS papers. The latest results from all analyses can be found on the ATLAS SUSY public results web page [2].

\section{Inclusive searches for gluino and first two generations of squarks}

Final states containing only jets and large missing transverse momentum. Five inclusive analysis channels characterised by increasing jet multiplicity from two to six are defined for this analysis [3]. In all cases isolated electrons or muons are vetoed. Each channel is used to construct between one and three signal regions (SRs) with "loose", "medium", or "tight" selections distinguished by requirements placed on $E_{\mathrm{T}}^{\mathrm{miss}} / m_{\mathrm{eff}}$ and $m_{\mathrm{eff}}$ (incl.), where $m_{\mathrm{eff}}=\sum_{i=1}^{N_{\text {lep }}} p_{T}^{l_{i}}+\sum_{j=1}^{N_{\text {jet }}} p_{T}^{\text {jet }}+E_{\mathrm{T}}^{\mathrm{miss}}$, and the sum runs over all jets with $p_{\mathrm{T}}>30 \mathrm{GeV}$ in the event. The dominant background sources are: $\mathrm{W}+\mathrm{jets}, \mathrm{Z}+\mathrm{jets}$, top quark pairs, single top quarks, and multiple jets. The production of semileptonically decaying dibosons is a small component $(<13 \%)$ of the total background and is estimated with MC simulated data normalised to theoretical cross-section predictions. 
Good agreement was seen between the numbers of events observed in the data and the numbers of events expected from SM processes. In the MSUGRA/CMSSM models, values of $m_{1 / 2}<340$ $\mathrm{GeV}$ are excluded at the $95 \%$ confidence level for $m_{0}<6 \mathrm{TeV}$ and $m_{1 / 2}<800 \mathrm{GeV}$ for low $m_{0}$. Equal mass squarks and gluinos are excluded below $1800 \mathrm{GeV}$ in this scenario (see Figure 1 left plot, violet line).

Final states with large jet multiplicities and missing transverse momentum. The event selection requires missing transverse momentum, no isolated electrons or muons, and from 7 to $\geqslant 10$ jets. The sensitivity of the search is enhanced by considering the number of $b$-tagged jets and the scalar sum of masses of large-radius $(R=1.0)$ jets in an event [4].

Nineteen signal regions are defined. Within the multi-jet + flavour stream the seven signal regions defined with higher jet $p_{\mathrm{T}}$ threshold $p_{T}^{\min }=50 \mathrm{GeV}$ are mutually disjoint. The same is true for the six signal regions defined with the threshold of $80 \mathrm{GeV}$. The multi-jet $+M_{J}^{\sum}\left(M_{J}^{\sum}\right.$ is the sum of the masses $m_{j}^{R=1.0}$ of the composite jets) stream has six inclusive signal regions; for example an event which has at least ten $R=0.4$ jets with $p_{T}>50 \mathrm{GeV}, M_{J}^{\sum}>420 \mathrm{GeV}$ and $E_{\mathrm{T}}^{\text {miss }} / \sqrt{H_{\mathrm{T}}}>4$ $\mathrm{GeV}^{1 / 2}\left(H_{\mathrm{T}}\right.$ is scalar sum of the $p_{\mathrm{T}}$ of the lepton(s) and jets) will be found in all six multi-jet $+M_{J}^{\sum}$ regions. The dominant backgrounds are multi-jet production, including purely strong interaction processes and fully hadronic decays of $t \bar{t}$, and hadronic decays of $\mathrm{W}$ and $\mathrm{Z}$ bosons in association with jets; semi- and fully-leptonic decays of $t \bar{t}$; and leptonically decaying $\mathrm{W}$ or $\mathrm{Z}$ bosons produced in association with jets. Non-fully-hadronic $t \bar{t}$, and events with leptonic decays of $\mathrm{W}$ and $\mathrm{Z}$ bosons are collectively referred to as leptonic backgrounds.

In the absence of significant discrepancies, exclusion limits at 95\% CL are set in the context of several simplified supersymmetric models and a mSUGRA/CMSSM model. Figure 1 left (blue lines) shows the exclusion limits in this model, where, for large universal scalar mass $m_{0}$, gluino masses smaller than $1.1 \mathrm{TeV}$ are excluded at 95\% CL. The right plot on Figure 1 (blue line) shows in the framework of a simplified model that gluino masses smaller than $1.1 \mathrm{TeV}$ for neutralino masses below $350 \mathrm{GeV}$ are excluded.

Final states with isolated leptons, jets and missing transverse momentum. Search for final states containing at least one isolated lepton (electron or muon), jets, with or without b-jet requirements, and large missing transverse momentum were also performed. The kinematic reach of the analysis is extended to soft leptons to increase the sensitivity to supersymmetric spectra with small mass splitting [5].

The soft single-lepton signal regions require one or two b-jets. A further subdvision is made by varying the requirement on the $E_{\mathrm{T}}^{\text {miss }}$ and, in the soft single-lepton two b-jet case, on $m_{C T}$ and $H_{T, 2}$ (where $m_{C T}^{2}\left(v_{1}, v_{2}\right)=\left[E_{T}\left(v_{1}\right)+E_{T}\left(v_{2}\right)\right]^{2}-\left[\overrightarrow{p_{T}}\left(v_{1}\right)-\overrightarrow{p_{T}}\left(v_{2}\right)\right]^{2}$ [12] and $\mathrm{H}_{T, 2}$ is the scalar sum of the $p_{\mathrm{T}}$ of all signal jets excluding the two leading jets). A veto is placed on the presence of a second lepton (in the single-lepton channels) or of a third lepton (in the soft dimuon case). In the hard single-lepton channels, two sets of requirements are optimized for each jet multiplicity: one inclusive signal region optimized for the discovery reach which can also be used to place modelindependent limits, and one signal region which is binned in $m_{\mathrm{eff}}\left(\mathrm{incl}\right.$.) or $E_{\mathrm{T}}^{\mathrm{miss}}$ in order to exploit the expected signal shape when placing model-dependent limits. The latter signal regions are made orthogonal in jet multiplicity from one another to allow their statistical combination and have more fine-grained $E_{\mathrm{T}}^{\text {miss }}, m_{\mathrm{T}}$ and $m_{\text {eff }}$ (incl.) requirements than in a single overall signal region. The main 
sources of SM background in the signal regions are $t \bar{t}$ and $\mathrm{W}+$ jets events, in which there is at least one genuine lepton originating from a $\mathrm{W}$ decay.

Observations are in general in good agreement with the Standard Model background expectations, although a non-significant excess (at $2.3 \sigma$ ) is seen in the soft dimuon channel. The limits in the $m_{0}-m_{1 / 2}$ MSUGRA/CMSSM plane obtained from the hard-lepton signal regions are shown in Figure 1 left plot (red lines). At large values of $m_{0}$, this analysis is able to exclude a gluino mass of up to $1.2 \mathrm{TeV}$.
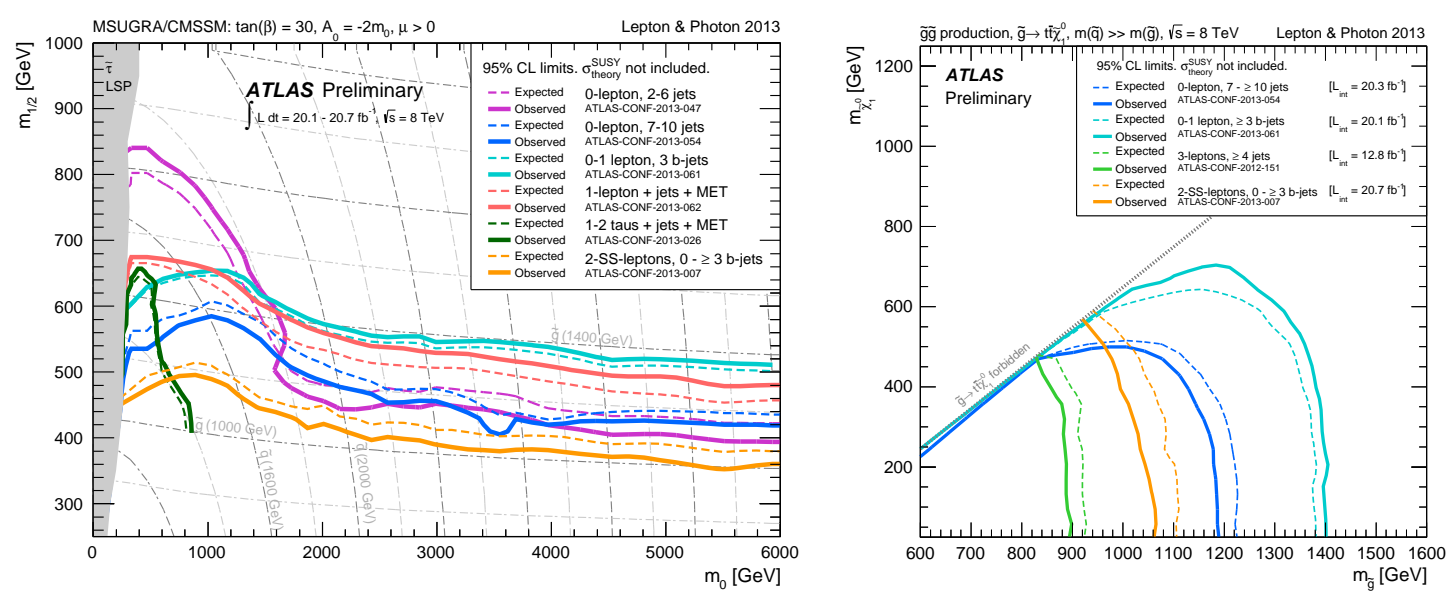

Figure 1: Left plot shows the exclusion limits at 95\% CL for $8 \mathrm{TeV}$ analyses in the $\left(m_{0}, m_{1 / 2}\right)$ plane for the MSUGRA/CMSSM model with the remaining parameters set to $\tan (\beta)=30, \mathrm{~A}_{0}=-2 m_{0}, \mu>0$. Part of the model plane accommodates a lightest neutral scalar Higgs boson mass of $125 \mathrm{GeV}$. Right plot shows the exclusion limits at 95\% CL for $8 \mathrm{TeV}$ analyses in the $\left.\left(m_{\tilde{g}}, m_{\tilde{\chi}_{1}^{0}}\right)\right)$ plane for the Gtt simplified model where a pair of gluinos decays promptly via off-shell stop to four top quarks and two lightest neutralinos (LSP).

\section{Search for direct third generation squark pair production}

Final states with missing transverse momentum and two b-jets. The lightest bottom and top squarks ( $\tilde{b}_{1}$ and $\tilde{t}_{1}$ respectively) are searched for in a final state with large missing transverse momentum and two jets identified as originating from a b-quark [6].

Two signal regions are defined within current analysis based on the $m_{C T}$ variable. Signal region A (SRA) requires two b-tagged leading jets, events are rejected if any further central $(|\eta|<2.8)$ jets are found with $p_{T}>50 \mathrm{GeV}$. Signal region B (SRB) selects final state events with a high- $p_{T}$ jet produced as initial state radiation recoiling against the squark pair system. High thresholds on the leading jet and on the missing transverse momentum, which are required to be almost back-to-back in $\phi$, are imposed. The leading jet is required to be not b-tagged and two additional jets are required to be b-tagged. The dominant SM background processes in the signal regions are the production of $\mathrm{W}$ or $\mathrm{Z}$ boson in association with heavy flavour jets ( $\mathrm{W}+\mathrm{hf}$ and $\mathrm{Z}+\mathrm{hf}$ ) and the production of top quarks.

The results are in agreement with SM predictions for backgrounds. For stop pair production decaying only into $b \tilde{\chi}_{1}^{ \pm}$, stop masses up to $580 \mathrm{GeV}(440 \mathrm{GeV})$ are excluded for $\Delta m=m_{\tilde{\chi}_{1}^{ \pm}}-m_{\tilde{\chi}_{1}^{0}}=$ 
$5 \mathrm{GeV}(20 \mathrm{GeV})$ and for $m_{\tilde{\chi}_{1}^{0}}=100 \mathrm{GeV}$. For $\Delta m=5 \mathrm{GeV}(20 \mathrm{GeV})$, neutralino masses up to 250 $\mathrm{GeV}(220 \mathrm{GeV})$ are excluded for $m_{\tilde{t}_{1}}=450 \mathrm{GeV}$ (see Figure 2).

Final states with one isolated lepton, jets, and missing transverse momentum. Direct stop quark pair production is searched for in final states with one isolated electron or muon, jets, and missing transverse momentum in proton-proton collisions at a centre-of-mass energy of $8 \mathrm{TeV}$ [7].

Six signal regions (SRs) are defined in order to optimize the sensitivity for different stop and LSP masses, as well as the two considered stop decay scenarios. Three signal regions have been optimized for the $\tilde{t}_{1} \rightarrow b+\tilde{\chi}_{1}^{ \pm}$decay scenario, and three signal regions optimized for the $\tilde{t}_{1} \rightarrow t+\tilde{\chi}_{1}^{0}$ decay scenario. The dominant background in all signal regions arises from dileptonic $t \bar{t}$ events in which one of the leptons is not identified, is outside the detector acceptance, or is a hadronically decaying $\tau$ lepton.

No significant excess of events above the rate predicted by the Standard Model is observed. Figure 2 shows that in the $\tilde{t}_{1} \rightarrow t+\tilde{\chi}_{1}^{0}$ decay scenario, top squark masses between 200 and 610 $\mathrm{GeV}$ are excluded at $95 \%$ confidence level for massless LSPs, while top squark masses around 500 $\mathrm{GeV}$ are excluded for LSP masses up to $250 \mathrm{GeV}$. In the $\tilde{t}_{1} \rightarrow b+\tilde{\chi}_{1}^{ \pm}$decay scenario, top squark masses up to $410 \mathrm{GeV}$ are excluded for massless LSPs in the case of a chargino with a mass of 150 $\mathrm{GeV}$.
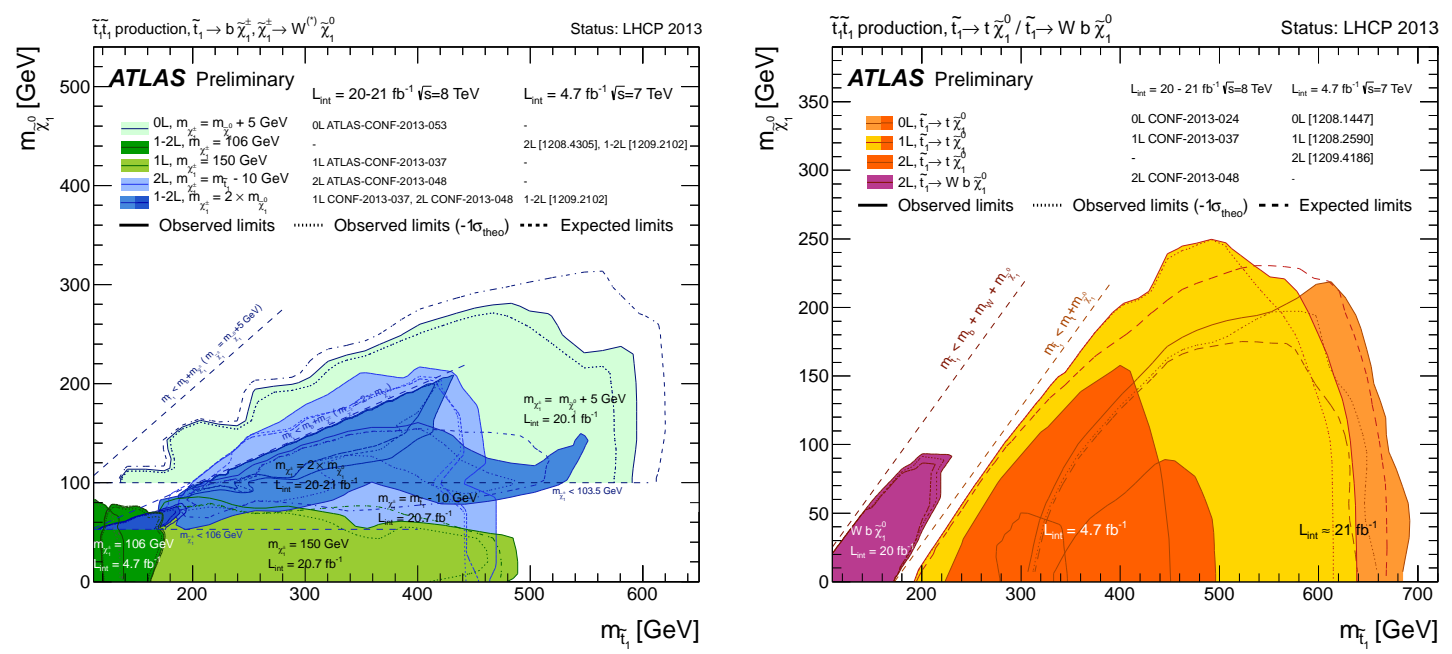

Figure 2: Summary of the dedicated ATLAS searches for top squark (stop) pair production based on 20 $\mathrm{fb}^{-1}$ of pp collision data taken at $\sqrt{s}=8 \mathrm{TeV}$ and $4.7 \mathrm{fb}^{-1}$ of pp collision data taken at $\sqrt{s}=7 \mathrm{TeV}$. Exclusion limits at $95 \% \mathrm{CL}$ are shown in the $\left(\tilde{t}_{1}-\tilde{\chi}_{1}^{0}\right)$ mass plane. The dashed and solid lines show the expected and observed limits, respectively, including all uncertainties except the theoretical signal cross section uncertainty (PDF and scale). The dotted lines represent the results obtained when reducing the nominal signal cross section by $1 \sigma$ of its theoretical uncertainty.

\section{Searches for the electroweak production of pairs of sleptons or charginos}

Final states with two opposite-sign leptons, missing transverse momentum and no jets. The searches summarized in this section target the following four scenarios that produce final states with two oppositely-charged leptons (electrons or muons) and missing transverse momentum [8]. 
Five signal regions (SRs) are defined in this analysis. The first two are designed to provide sensitivity to sleptons either through direct production or in chargino decays. The other three are designed to provide sensitivity to chargino and neutralino-pair production followed by on-shell W decays. SM background is dominated by events with two leptonically-decaying $\mathrm{W}$ bosons coming from WW diboson and top production. Another significant source of background, in the sameflavour channel, is $\mathrm{ZV}$ production (where $\mathrm{V}=\mathrm{Z}$ or $\mathrm{W}$ ).

No significant excesses over the Standard Model predictions are observed. The best sensitivity for direct chargino pair production with charginos decaying into $\mathrm{W}$ bosons is obtained for the $\left(m_{\tilde{\chi}_{1}^{ \pm}}, m_{\tilde{\chi}_{1}^{0}}\right)=(100,0) \mathrm{GeV}$ mass point, where a signal with a cross-section larger than 1.8 times the SUSY cross section is excluded at 95\% CL.

Final states with three leptons and missing transverse momentum. This section presents a search with the ATLAS detector for the direct production of charginos and neutralinos decaying to a final state with three leptons (electrons or muons) and missing transverse momentum, the latter originating from the two undetected LSPs and the neutrinos [9].

"Loose", "medium" and "tight" signal regions are defined for the six Z-depleted and Z-rich regions: three "Z-depleted" regions, with no same-flavour opposite-sign (SFOS) pairs having an invariant mass within $10 \mathrm{GeV}$ of the Z-boson mass; and three "Z-enriched" regions. The SM background is dominated by events from the $t \bar{t}$, single top quark, $\mathrm{V}+\mathrm{jets}$, di, tribosons and $t \bar{t}+\mathrm{V}$ production processes.

No significant excess of events is found in data above SM expectations. For the simplified SUSY models with intermediate slepton decays, degenerate $\tilde{\chi}_{1}^{ \pm}$and $\tilde{\chi}_{2}^{0}$ masses up to $600 \mathrm{GeV}$ are excluded for large mass differences with the $\tilde{\chi}_{1}^{0}$. For the simplified SUSY models with gauge boson decays, degenerate $\tilde{\chi}_{1}^{ \pm}$and $\tilde{\chi}_{2}^{0}$ masses up to $315 \mathrm{GeV}$ are excluded for large mass differences with the $\tilde{\chi}_{1}^{0}$.

Final states with at least two hadronically decaying taus and missing transverse momentum. Final states containing at least two hadronically decaying taus are considered in this search. At least one tau pair is required to have opposite sign (OS) [10]. Events with additional light leptons (electrons or muons) are vetoed to allow for a statistical combination with similar searches using light leptons.

Two signal regions are defined: SR OS- $m_{T 2}$ (a jet veto is applied, $E_{\mathrm{T}}^{\text {miss }}>40 \mathrm{GeV}$ and $m_{T 2}>$ $90 \mathrm{GeV}$, where $\left.m_{T 2}=\min _{q_{T}}\left[\max \left(m_{\mathrm{T}}\left(p_{T}^{l 1}, q_{T}\right), m_{\mathrm{T}}\left(p_{T}^{l 2}, p_{\mathrm{T}}^{\text {miss }}-q_{T}\right)\right)\right][13,14]\right)$ and SR OS- $m_{T 2}$-nobjet (b-jet veto is applied, $E_{\mathrm{T}}^{\text {miss }}>40 \mathrm{GeV}$ and $m_{T 2}>100 \mathrm{GeV}$ ). The main SM background processes contributing to the selected final states are multi-jet production and $\mathrm{W}$ bosons produced in association with jets.

Good agreement between data and SM expectations is observed in all signal regions. For Simplified Models, chargino masses up to $350 \mathrm{GeV}$ are excluded for a massless lightest neutralino in the scenario of direct production of wino-like chargino pairs decaying into the lightest neutralino via an intermediate on-shell tau slepton. In the case of pair production of degenerate charginos and next-to-lightest neutralinos, masses up to 330 (300) GeV are excluded for lightest neutralino masses below 50 (100) GeV.

Final states with four or more leptons. A search for new physics was performed based on events with four or more isolated leptons [11]. The term "lepton" refers to electrons, muons and 
taus, and the term "light lepton" here refers to electrons and muons only, including those from leptonic tau decays. Here, four lepton combinations with at least three light leptons are considered.

Two signal regions are defined for each allowed tau multiplicity: one vetoing $\mathrm{Z}$ candidates and one requiring $\mathrm{Z}$ candidates. The distinction is based on the selection of events with pairs, triplets or quadruplets of light leptons with an invariant mass inside the [81.2, 101.2] GeV interval. The dominant SM process passing this selection is the ZZ production, where both Z-bosons decay leptonically.

No significant excess of events is found in the data. Observed 95\% CL limits on the visible cross-sections are placed at $0.23 \mathrm{fb}$ and $0.18 \mathrm{fb}(0.50 \mathrm{fb})$ in the $Z$ veto ( $Z$ request $) \geqslant 4 l \geqslant 0 \tau$ signal regions, and at $0.36 \mathrm{fb}(0.31 \mathrm{fb})$ in the $Z$ veto $(Z$ request $)=3 l \geqslant 1 \tau$ signal regions used in this analysis.

\section{Conclusion}

The most recent analysis based on more than $20.7 \mathrm{fb}^{-1}$ of proton-proton collision data recorded by the ATLAS detector at the LHC at $\sqrt{s}=8 \mathrm{TeV}$. No significant excess of events is found in the data. Observed 95\% CL limits on the visible cross-sections for the various signal regions are set. The null result is interpreted in a range of simplified models, and in constrained models such as mSUGRA/MSSM, GMSB. Limits on masses of sparticles such as gluinos, squarks, neutrolinos, charginos, slepton and squarks of the third generation are obtained within the considered SUSY models.

\section{References}

[1] ATLAS Collaboration, JINST 3 (2008), S08003.

[2] https://twiki.cern.ch/twiki/bin/view/AtlasPublic/SupersymmetryPublicResults.

[3] ATLAS Collaboration, ATLAS-CONF-2013-047. http://cds.cern.ch/record/1547563.

[4] ATLAS Collaboration, ATLAS-CONF-2013-054. http://cds.cern.ch/record/1547571.

[5] ATLAS Collaboration, ATLAS-CONF-2013-062. http://cds.cern.ch/record/1557779.

[6] ATLAS Collaboration, ATLAS-CONF-2013-053. http://cds.cern.ch/record/1547570.

[7] ATLAS Collaboration, ATLAS-CONF-2013-037. https://cds.cern.ch/record/1532431.

[8] ATLAS Collaboration, ATLAS-CONF-2013-049. https://cds.cern.ch/record/1547565.

[9] ATLAS Collaboration, ATLAS-CONF-2013-035. https://cds.cern.ch/record/1532426.

[10] ATLAS Collaboration, ATLAS-CONF-2013-028. https://cds.cern.ch/record/1525889.

[11] ATLAS Collaboration, ATLAS-CONF-2013-036. https://cds.cern.ch/record/1532429.

[12] D.R. Tovey, On measuring the masses of pair-produced semi-invisibly decaying particles at hadron colliders, JHEP 0804 (2008) 034, [hep-ph/ 0802 . 2879].

[13] C.G. Lester and D.J. Summers, Measuring masses of semiinvisibly decaying particles pair produced at hadron colliders, Phys. Lett. B463 (1999) 99, [arXiv: hep-ph/ 9906349 ].

[14] A. Barr, C. Lester, and P. Stephens, $m($ T2) : The Truth behind the glamour, J. Phys. G29 (2003) 2343, [arXiv: hep-ph/0304226]. 